\title{
Cavity control of nonlinear phononics
}

\author{
Dominik M. Juraschek $\odot,{ }^{1, *}, \dagger$ Tomáš Neuman $\odot,{ }^{1,},{ }^{*}$ Johannes Flick $\odot,{ }^{1,2}$ and Prineha Narang $\oplus^{1, \ddagger}$ \\ ${ }^{1}$ Harvard John A. Paulson School of Engineering and Applied Sciences, Harvard University, Cambridge, Massachusetts 02138, USA \\ ${ }^{2}$ Center for Computational Quantum Physics, Flatiron Institute, New York, New York 10010, USA
}

(Received 25 February 2020; revised 14 April 2021; accepted 28 July 2021; published 16 August 2021)

\begin{abstract}
Nonlinear interactions between phonon modes govern the behavior of vibrationally highly excited solids and molecules. Here we demonstrate theoretically control over the redistribution of energy from a highly excited coherent infrared-active phonon state into other vibrational degrees of freedom of the system using optical cavities. Specifically, we tune the polaritonic splitting of an infrared-active mode in the cavity so that it is pushed into resonance with other phonon modes of the system. This technique makes it possible to tune the efficiency of energy redistribution and even to change the underlying scattering mechanism, which possibly enables the creation of phonon-induced states in a broad scale of materials that do not naturally exhibit the required resonance conditions.
\end{abstract}

DOI: 10.1103/PhysRevResearch.3.L032046

Strong vibrational excitations of solids are able to modify electronic correlations in a material and induce emergent states of matter that do not exist in equilibrium [1,2]. Phenomena based on nonlinear interactions between phonon modes have, most prominently, lead to light-induced superconductivity [3-10], and control over ferroelectricity [11-16] and magnetism [17-33] in recent years. These phenomena are based on the energy redistribution from a coherently excited infrared (IR)-active mode to Raman-active ones that couple to the electronic system through transient modulations of the crystal structure and the electron-phonon coupling. The efficiency of the energy transfer is, however, often hindered due to a frequency mismatch of the phonon modes participating in the interaction. At the same time, developments in the field of strong light-matter coupling have made it possible to engineer optical properties of materials in cavities. An electromagnetic mode sustained in the cavity hybridizes with optically active states of the material to form polariton states, as depicted in Fig. 1(a). This polaritonic engineering has been used to modify electronic excitations, such as plasmons and excitons [34-36] and to modulate the chemical landscape of molecules [37-41], and various studies suggest an influence of strong light-matter coupling on superconductivity [42-45].

Here, we synthesize the physics of nonlinear phononics and strong light-matter coupling to overcome frequency mismatches in phononic scattering processes. The goal of this study is to demonstrate a proof-of-principle technique that

\footnotetext{
*These authors contributed equally to this work.

†juraschek@seas.harvard.edu

"prineha@seas.harvard.edu
}

Published by the American Physical Society under the terms of the Creative Commons Attribution 4.0 International license. Further distribution of this work must maintain attribution to the author(s) and the published article's title, journal citation, and DOI. allows us to achieve advanced tuning capabilities for coherent nonlinear vibrational dynamics. We sketch the principle of the technique for the example of a parametric downconversion process in Figs. 1(b) and 1(c). In Fig. 1(b), an IR-active mode (eigenfrequency $\Omega_{\mathrm{IR}}$ ) is out of resonance with two Raman-active modes (eigenfrequencies $\Omega_{R}$ ), preventing efficient coupling between them. By tuning the level splitting of the IR-active mode in a terahertz-frequency cavity in Fig. 1(c), one of the phonon-polariton branches is shifted into resonance with the Raman-active modes, $\Omega_{-}=2 \Omega_{\mathrm{R}}$, enabling the redistribution of energy between them.

Nonlinear phononic processes. We begin by analyzing the dominant nonlinear phononic scattering processes between coherently excited IR-active modes and Raman-active ones, which we display in Table I. Essential features of

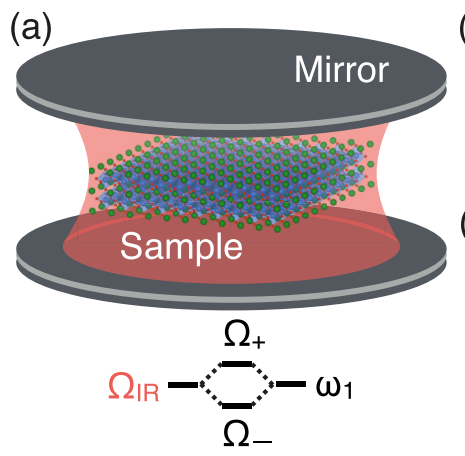

(b)

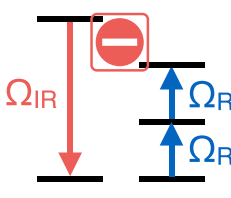

(c)

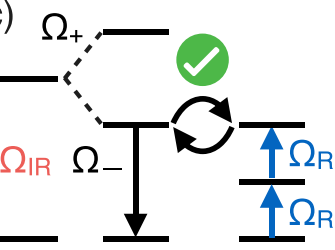

FIG. 1. Cavity-induced resonance in nonlinear phononics. (a) Schematic setup of a sample material in a terahertz cavity. An IR-active mode $\Omega_{\mathrm{IR}}$ and the fundamental cavity mode $\omega_{1}$ hybridize and form two phonon-polariton modes $\Omega_{+}$and $\Omega_{-}$. (b) Frequency mismatch between an IR-active and two Raman-active modes $2 \Omega_{\mathrm{R}}$ hinders the transfer of energy between them. (c) Polaritonic splitting tunes the energy of the lower branch into resonance with the Ramanactive modes. 
experimentally observed phenomena can be often be reduced to a few dominant nonlinear phonon couplings that are allowed by the symmetries of their irreducible representations $[10,46,47]$. The potential energy $V$, including the most prominent phonon couplings beyond the harmonic approximation, can be written as [46]

$$
\begin{aligned}
V\left(Q_{\mathrm{IR}}, Q_{\mathrm{R}}\right)= & \frac{\Omega_{\mathrm{IR}}^{2}}{2} Q_{\mathrm{IR}}^{2}+\frac{\Omega_{\mathrm{R}}^{2}}{2} Q_{\mathrm{R}}^{2} \\
& +c Q_{\mathrm{IR}}^{2} Q_{\mathrm{R}}+d Q_{\mathrm{IR}} Q_{\mathrm{R}}^{2}+e Q_{\mathrm{IR}}^{2} Q_{\mathrm{R}}^{2} .
\end{aligned}
$$

Here, $Q_{\mathrm{IR}}$ and $Q_{\mathrm{R}}$ are the normal mode coordinates (amplitudes) of the IR and Raman-active modes in $\AA \sqrt{\mathrm{amu}}$, where amu is the atomic mass unit. $c, d$, and $e$ are the phonon coupling coefficients in $\mathrm{meV} /(\AA \sqrt{\mathrm{amu}})^{n}$, where $n$ is the order of expansion in the phonon amplitudes. The coefficient $d$ is nonzero only in noncentrosymmetric (e.g., ferroelectric or polar) materials.

Leading nonlinear phononic mechanisms can be classified as those linear and squared in the Raman-active phonon amplitude. In the linear case, the square of the IR-active phonon amplitude $Q_{\mathrm{IR}}^{2}$ acts as an effective driving force on the Raman-active mode. If $Q_{\mathrm{IR}} \sim A_{\mathrm{IR}} \sin \left(\Omega_{\mathrm{IR}} t\right)$ is sinusoidal with a time-dependent amplitude $A_{\mathrm{IR}}$ then $Q_{\mathrm{IR}}^{2} \sim$ $A_{\mathrm{IR}}^{2}\left[1-2 \cos \left(2 \Omega_{\mathrm{IR}} t\right)\right]$ possesses a static component and one oscillating at twice the frequency. More generally, for finite linewidths, difference, and sum-frequency components $\Omega_{\mathrm{IR} 1} \mp \Omega_{\mathrm{IR} 2}$ appear. Depending on whether the IR-active mode has a higher or smaller frequency than the Ramanactive one, this coupling leads to either difference-frequency (DF) or sum-frequency ionic Raman scattering (SF-IRS), see Table I. The static force component in DF-IRS transiently distorts the crystal structure and it is held responsible for the enhancement of superconductivity in yttrium barium copper oxide [3,48-50], and for switching of ferroic order in a ferroelectric $[11,12,16]$ and in a magnet [26]. SF-IRS, in contrast, is a two-phonon absorption process resonant at $\Omega_{\mathrm{IR}}=\Omega_{\mathrm{R}} / 2$, transferring energy to higher-frequency Raman-active modes [51-55].

In the quadratic case, the IR-active phonon amplitude effectively modulates the Raman-active phonon frequency $\tilde{\Omega}_{\mathrm{R}}^{2}=\Omega_{\mathrm{R}}^{2}+2 d Q_{\mathrm{IR}}+2 e Q_{\mathrm{IR}}^{2}$. This modulation leads to a parametric amplification of the Raman-active mode, when either resonance condition $\Omega_{\mathrm{IR}}=2 \Omega_{\mathrm{R}}\left(Q_{\mathrm{IR}} Q_{\mathrm{R}}^{2}\right.$ coupling $)$ or $\Omega_{\mathrm{IR}}=\Omega_{\mathrm{R}} \sqrt{1+e\left(A_{\mathrm{IR}} / \Omega_{\mathrm{R}}\right)^{2}}\left(Q_{\mathrm{IR}}^{2} Q_{\mathrm{R}}^{2}\right.$ coupling $)$ are fulfilled, see Table I. Parametric amplification was suggested to enhance superconductivity through an enhancement of electron-phonon coupling [5-7,56], and can be used to generate squeezed phonon states $[57,58]$. The symmetry of the parametric coupling allows an IR-active mode, in addition to Raman-active modes, to also couple to silent phonon modes that are otherwise inaccessible for coherent excitation [59]. To demonstrate the principle of coherent control over nonlinear phonon dynamics, we will focus on the leading-order contributions from three-phonon processes in the remainder of the manuscript and set $e=0$.

Nonlinear phonon dynamics in a cavity. We now investigate how the redistribution of energy changes when we tune the cavity mode in and out of resonance with the IR-active mode in the geometry shown in Fig. 1(a). The coherent state of lightdriven phonons can be described by semiclassical oscillator equations, taking into account the light-matter interaction and the nonlinear phonon couplings [11,21-24,46-48,51,60-64]. The coupled equations of motion for the amplitudes of the IR-active, Raman-active, and fundamental cavity modes, $Q_{\mathrm{IR}}$, $Q_{\mathrm{R}}$, and $A_{1}$, can be written as

$$
\begin{gathered}
\ddot{A}_{1}+\kappa_{1} \dot{A}_{1}+\omega_{1}^{2} A_{1}=B_{1} E(t)+D_{1} \ddot{Q}_{\mathrm{IR}}, \\
\ddot{Q}_{\mathrm{IR}}+\kappa_{\mathrm{IR}} \dot{Q}_{\mathrm{IR}}+\partial_{Q_{\mathrm{IR}}} V=G_{1} A_{1}, \\
\ddot{Q}_{\mathrm{R}}+\kappa_{\mathrm{R}} \dot{Q}_{\mathrm{R}}+\partial_{Q_{\mathrm{R}}} V=0 .
\end{gathered}
$$

The fundamental cavity mode is excited by irradiating the mirrors of the cavity with a terahertz pulse, which in turn radiate into the cavity, coupling to the IR-active mode in the sample. For a detailed derivation of the equations, please see the Supplemental Material [65]. Here, $E(t)=E_{0} \exp \left\{-t^{2} /\left[2(\tau / \sqrt{8 \ln 2})^{2}\right]\right\} \cos \left(\omega_{0} t\right)$ is the electricfield component of the terahertz pulse, where $E_{0}$ is the peak electric field, $\omega_{0}$ is the center frequency, and $\tau$ is the full width at half maximum duration. Because of the small momentum of terahertz radiation compared to the crystal momentum, we can neglect any wave-vector dependence in the equations above. For the material specific parameters, we choose values typical for transition-metal oxides [11,22,23,25,46,48,62,63]. We set the coupling coefficients to $c=d=100 \mathrm{meV} /(\AA \sqrt{\mathrm{amu}})^{3}$, the mode effective charge of the IR-active mode to $Z_{\mathrm{IR}}=1$

TABLE I. Nonlinear phononic processes. From left to right: difference and sum-frequency ionic Raman scattering, and cubic and quarticorder parametric amplification. Shown are the terms of the lattice potential expansion in the IR and Raman-active phonon amplitudes $Q_{\mathrm{IR}}$ and $Q_{\mathrm{IR}}$ in Eq. (1), as well as the resonance conditions for their frequency components $\Omega_{\mathrm{IR}(1,2)}$ and $\Omega_{\mathrm{R}}$.

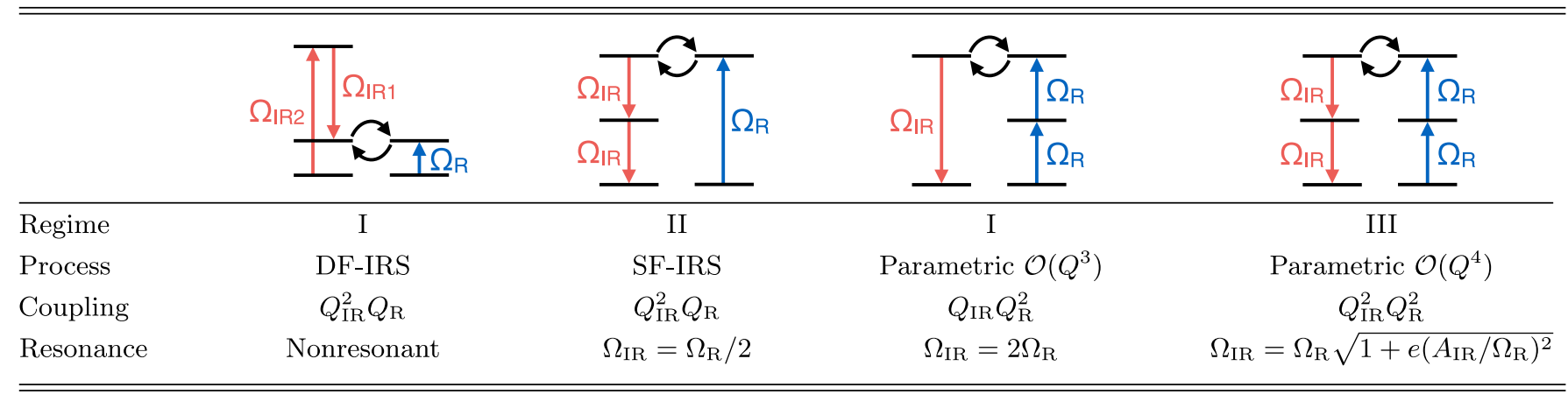



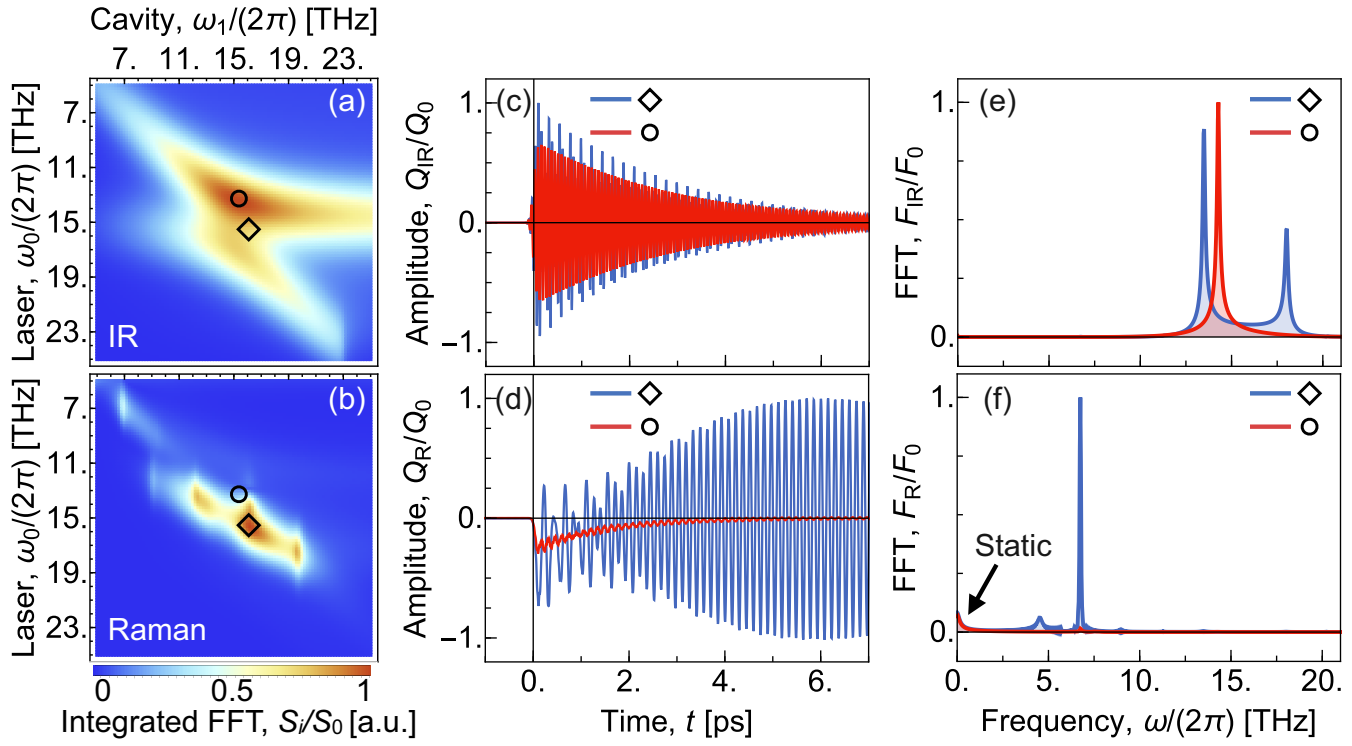

FIG. 2. Nonlinear phonon dynamics in regime I. (a,b) Normalized integrated spectral densities $S_{i} / S_{0}$ of the 15 THz IR $(i=$ IR) and 6.75 THz Raman-active $(i=\mathrm{R})$ phonon modes for different frequencies of the laser pulse $\omega_{0}$ and cavity mode $\omega_{1}$. The circle marks a point where the IR and Raman-active modes are out of resonance $\Omega_{\mathrm{IR}}>2 \Omega_{\mathrm{R}}$, which hinders the transfer of energy. The diamond marks the point, where the polariton splitting in the cavity tunes the IR and Raman-active modes into resonance $\Omega_{-}=2 \Omega_{\mathrm{R}}$, enabling parametric amplification. (c,d) Time evolution of the normalized phonon amplitudes, $Q_{i} / Q_{0}$, of the IR and Raman-active modes in and out of resonance. (e,f) Normalized FFT spectra, $F_{i} / F_{0}$, of the IR and Raman-active modes in and out of resonance. The black arrow points to the static zero-frequency response.

$\mathrm{amu}^{-1} e$ [66], where $e$ is the elementary charge, the volume of the unit cell to $V_{\mathrm{c}}=50 \AA^{3}$, and the phonon linewidths to $\kappa_{i}=0.05 \times \Omega_{i} /(2 \pi)$, where $i \in\{1, \mathrm{IR}, \mathrm{R}\}$. The coefficients $B_{1}, D_{1}$, and $G_{1}$ contain the light-matter and phonon coupling coefficients and are further dependent on the geometry of the system and the sample thickness. We specify them in the Supplementary Material [65]. We find that a sample thickness of $\Delta z=100 \mathrm{~nm}$ is sufficient to yield a significant splitting of the phonon-polariton branches ( $>20 \%$ of $\Omega_{\mathrm{IR}}$ ), while justifying the approximations made in the derivations of Eqs. (2) to (4). This splitting is sufficient to cover commonly occurring frequency mismatches in transition-metal oxides. We vary the frequencies of the terahertz pulse and the cavity mode as possible in an experiment, and look at the two regimes of three-phonon processes: First (I), $\Omega_{\mathrm{IR}}>\Omega_{R}$, for DF-IRS and cubic-order parametric amplification, and second (II), $\Omega_{\mathrm{IR}}<\Omega_{\mathrm{R}}$, for SF-IRS.

Results for regime I. We start with the example of a $15 \mathrm{THz}$ IR and a $6.75 \mathrm{THz}$ Raman-active mode that are detuned by $\Delta \Omega /(2 \pi)=1.5 \mathrm{THz}$ such that $\Omega_{\mathrm{IR}}-\Delta \Omega=2 \Omega_{\mathrm{R}}$. We show the response of the phonon modes to the excitation by a terahertz pulse with a peak electric field of $E_{0}=10 \mathrm{MV} / \mathrm{cm}$ and a duration of $\tau=250 \mathrm{fs}$ in Fig. 2. Figures 2(a) and 2(b) show maps of the integrated spectral densities, $S_{i}=\int_{0}^{\infty}\left|F_{i}\right|^{2} d \omega$, using fast Fourier transformation (FFT) spectra $F_{i}$ of the phonon amplitudes $Q_{i}$ that we obtain solving Eqs. (2) to (4). We plot the maps as a function of the pulse frequency $\omega_{0}$ and cavity frequency $\omega_{1}$. The values are normalized to the maxima of the respective phonon modes $S_{0}=\max \left[S_{i}(\omega)\right]$.

The integrated spectral density of the IR-active mode $S_{\mathrm{IR}}$, in Fig. 2(a) forms two continuous bands that exhibit avoided crossing, a clear signature of phonon-photon strong coupling.
$S_{\text {IR }}$ increases towards the center of the map, where $\omega_{1}$ approaches the value of $\Omega_{\mathrm{IR}}$, as the IR-active mode couples resonantly to the cavity mode, but reduces at the very center of the map, where the two modes hybridize and the energy is shared between the two phonon-polariton branches. In contrast, the integrated spectral density of the Raman-active mode, $S_{R}$, in Fig. 2(b) exhibits a sharp peak at $\omega_{0} /(2 \pi)=15.6 \mathrm{THz}$ and $\omega_{1} /(2 \pi)=16.2 \mathrm{THz}$ and two sidepeaks. To illustrate the effect of resonance tuning, we select two distinct points in these frequency maps, at which the response of the IR-active mode is roughly equivalent in magnitude, but the response of the Raman-active mode differs drastically. The diamond symbol marks the point where the integrated spectral density of the Raman-active mode is maximal $S_{\mathrm{R}} / S_{0}=1$ and the circle marks a point with a much smaller $S_{\mathrm{R}} / S_{0}$ value, which is here chosen at $\omega_{0} /(2 \pi)=15.4$ $\mathrm{THz}$ and $\omega_{1} /(2 \pi)=13.4 \mathrm{THz}$.

For these two points, we show the time evolutions of the phonon amplitudes $Q_{i}$ in Figs. 2(c) and 2(d), and their corresponding FFT spectra $F_{i}$ in Figs. 2(e) and 2(f). The values are again normalized to the respective maxima $Q_{0}=\max \left[Q_{i}(t)\right]$ and $F_{0}=\max \left[F_{i}(t)\right]$. The IR-active mode in Fig. 2(c) shows the typical evolution of a resonantly driven and slowly decaying phonon mode at the out-of-resonance point, while an additional frequency component appears at the in-resonance point. This can be understood from looking at the FFT spectrum in Fig. 2(e). Out of resonance, the spectrum shows just a single peak that is slightly detuned from the eigenfrequency $\Omega_{\mathrm{IR}}$, but in resonance, the spectrum shows two frequency components that correspond to the contributions of the IRactive mode to the upper and lower phonon-polariton branches that arise from its hybridization with the cavity mode. The 

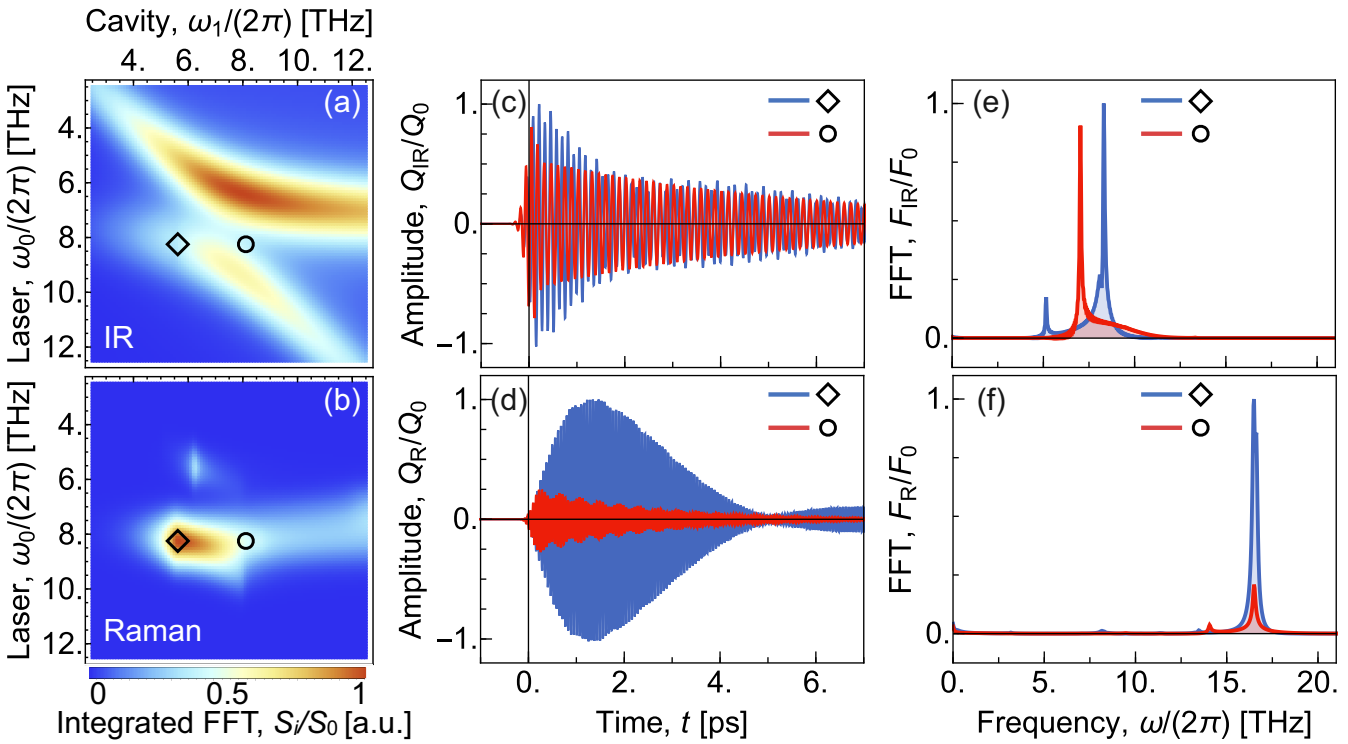

FIG. 3. Nonlinear phonon dynamics in regime II. (a,b) Normalized integrated spectral densities $S_{i} / S_{0}$ of the $7.5 \mathrm{THz}$ IR $(i=$ IR) and 16.5 THz Raman-active $(i=\mathrm{R})$ phonon modes for different frequencies of the laser pulse $\omega_{0}$ and cavity mode $\omega_{1}$. The circle marks a point where the IR and Raman-active modes are out of resonance $\Omega_{\mathrm{IR}}<\Omega_{\mathrm{R}} / 2$, which hinders the transfer of energy. The diamond marks the point where the polariton splitting in the cavity tunes the IR and Raman-active modes into resonance $\Omega_{+}=\Omega_{\mathrm{R}} / 2$, enabling parametric amplification. (c,d) Time evolution of the normalized phonon amplitudes $Q_{i} / Q_{0}$ of the IR and Raman-active modes in and out of resonance. (e,f) Normalized FFT spectra, $F_{i} / F_{0}$, of the IR and Raman-active modes in and out of resonance.

effect of this hybridization on the Raman-active mode can be seen in Fig. 2(d): out of resonance, the phonon amplitude exhibits a transient shift of its equilibrium position and only small vibrational amplitude, a typical feature of DF-IRS. In resonance, a large-amplitude oscillation builds up over time through the cubic-order parametric downconversion process. This reflects in the FFT spectrum in Fig. 2(f), where out of resonance there is only a small static component at zero frequency, but in resonance, a large peak at the eigenfrequency of the Raman-active mode appears. Comparing the two FFT spectra, it becomes clear that the frequency of the lower phonon-polariton branch is shifted into resonance with the Raman-active mode $\Omega_{-}=2 \Omega_{\mathrm{R}}$ enabling parametric downconversion.

Results for regime II. We next investigate the case of a $7.5 \mathrm{THz}$ IR and a $16.5 \mathrm{THz}$ Raman-active mode that are detuned by $\Delta \Omega /(2 \pi)=0.75 \mathrm{THz}$ such that $2\left(\Omega_{\mathrm{IR}}-\Delta \Omega\right)=$ $\Omega_{\mathrm{R}}$. We show the response of the phonon modes to an excitation by a terahertz pulse with $E_{0}=7 \mathrm{MV} / \mathrm{cm}$ and $\tau=500$ fs in Fig. 3, which is structured analogously to Fig. 2. As in regime I, the integrated spectral density of the IR-active mode $S_{\text {IR }}$ in Fig. 3(a) shows avoided crossing as a result of phononphoton strong coupling. $S_{\mathrm{R}}$ in Fig. 3(b) in turn exhibits a peak around $\omega_{0} /(2 \pi)=8.2 \mathrm{THz}$ and $\omega_{1} /(2 \pi)=5.7 \mathrm{THz}$. We again select the in-resonance point, as well as one out-ofresonance point, which is here chosen at $\omega_{0} /(2 \pi)=6.4 \mathrm{THz}$ and $\omega_{1} /(2 \pi)=8.1 \mathrm{THz}$. We find that the $d Q_{\mathrm{IR}} Q_{\mathrm{R}}^{2}$ coupling is negligible in this regime, as the dynamics remain unchanged when we set $d=0$. The features of the Raman-active mode response are therefore determined entirely by SF-IRS.

We show the time evolutions of the phonon amplitudes $Q_{i}$ in Figs. 3(c) and 3(d), and their corresponding FFT spectra
$F_{i}$ in Figs. 3(e) and 3(f). In Fig. 3(c), the IR-active phonon shows similar behavior both at the out-of and in-resonance points, a resonant build-up of the phonon amplitude, which then drops as it transfers energy to the Raman-active mode. In resonance, and additional frequency component emerges, which can be seen more prominently in the FFT spectrum in Fig. 3(e), and which again correspond to the upper and lower phonon-polariton branches. The amount of transferred energy strongly differs for the two cases, as can be seen from the response of the Raman-active phonon in in Fig. 3(d). The Raman-active phonon amplitude $Q_{\mathrm{R}}$ is oscillating with small amplitude at the out-of-resonance point and gets strongly enhanced when the cavity tunes the IR-active modes into resonance with the Raman-active one. Comparing the two FFT spectra, it becomes clear that the frequency of the upper phonon-polariton branch is shifted into resonance with the Raman-active mode $\Omega_{+}=\Omega_{\mathrm{R}} / 2$ enabling sum-frequency excitation. The increase of $Q_{\mathrm{R}}$ by a factor of 4 in this case increases the efficiency of energy redistribution by a factor of 16 , as the energy scales quadratically with the phonon amplitude.

Concluding remarks. We demonstrated a technique that allows us to tune the energy redistribution from highly excited IR-active into Raman-active modes on demand. This effect is reciprocal and can also be applied to cases, in which an energy transfer from Raman-active modes into the different branches of the phonon polariton is desired. The principle of the technique is general and can straightforwardly be applied to the coupling of IR-active phonons to other collective excitations. In particular, we anticipate that the technique may accelerate the utilization of nonequilibrium states arising from nonlinear coupling of phonon modes to electronic and superconducting 
order-parameter excitations [10,67-70], to magnetic and ferroelectric degrees of freedom [71-73], and to exotic photonic states [74].

Acknowledgments. We are grateful to Morgan Trassin, Denis Golež, Antoine Georges, and Eugene Demler for useful discussions. This work was supported by the Swiss National Science Foundation (SNSF) under project ID 184259, the DARPA DRINQS Program under Award No. D18AC00014, and Harvard University. T.N. acknowledges the Photonics at Thermodynamic Limits Energy Frontier Research Center funded by the US DoE under Award No. DESC0019140. J.F. acknowledges financial support from the Deutsche Forschungsgemeinschaft (DFG) under Contract No. FL 997/1-1. P.N. is a Moore Inventor Fellow supported by the Gordon and Betty Moore Foundation and is a CIFAR Azrieli Global Scholar.
[1] M. Först, C. Manzoni, S. Kaiser, Y. Tomioka, Y. Tokura, R. Merlin, and A. Cavalleri, Nonlinear phononics as an ultrafast route to lattice control, Nat. Phys. 7, 854 (2011).

[2] D. N. Basov, R. D. Averitt, and D. Hsieh, Towards properties on demand in quantum materials, Nat. Mater. 16, 1077 (2017).

[3] R. Mankowsky, A. Subedi, M. Först, S. O. Mariager, M. Chollet, H. T. Lemke, J. S. Robinson, J. M. Glownia, M. P. Minitti, A. Frano, M. Fechner, N. A. Spaldin, T. Loew, B. Keimer, A. Georges, and A. Cavalleri, Nonlinear lattice dynamics as a basis for enhanced superconductivity in $\mathrm{YBa}_{2} \mathrm{Cu}_{3} \mathrm{O}_{6.5}$, Nature (London) 516, 71 (2014).

[4] M. Mitrano, A. Cantaluppi, D. Nicoletti, S. Kaiser, A. Perucchi, S. Lupi, P. Di Pietro, D. Pontiroli, M. Riccò, S. R. Clark, D. Jaksch, and A. Cavalleri, Possible light-induced superconductivity in $\mathrm{K}_{3} \mathrm{C}_{60}$ at high temperature, Nature (London) 530, 461 (2016).

[5] M. Knap, M. Babadi, G. Refael, I. Martin, and E. Demler, Dynamical Cooper pairing in nonequilibrium electron-phonon systems, Phys. Rev. B 94, 214504 (2016).

[6] A. Komnik and M. Thorwart, BCS theory of driven superconductivity, Eur. Phys. J. B 89, 244 (2016).

[7] M. Babadi, M. Knap, I. Martin, G. Refael, and E. Demler, Theory of parametrically amplified electron-phonon superconductivity, Phys. Rev. B 96, 014512 (2017).

[8] D. M. Kennes, E. Y. Wilner, D. R. Reichman, and A. J. Millis, Transient superconductivity from electronic squeezing of optically pumped phonons, Nat. Phys. 13, 479 (2017).

[9] A. Cantaluppi, M. Buzzi, G. Jotzu, D. Nicoletti, M. Mitrano, D. Pontiroli, M. Riccò, A. Perucchi, P. Di Pietro, and A. Cavalleri, Pressure tuning of light-induced superconductivity in $\mathrm{K}_{3} \mathrm{C}_{60}$, Nat. Phys. 14, 837 (2018).

[10] B. Liu, M. Först, M. Fechner, D. Nicoletti, J. Porras, T. Loew, B. Keimer, and A. Cavalleri, Two pump frequency resonances for light-induced superconductivity in $\mathrm{YBa}_{2} \mathrm{Cu}_{3} \mathrm{O}_{6.5}$, Phys. Rev. X 10, 011053 (2020)

[11] A. Subedi, Proposal for ultrafast switching of ferroelectrics using midinfrared pulses, Phys. Rev. B 92, 214303 (2015).

[12] R. Mankowsky, A. von Hoegen, M. Först, and A. Cavalleri, Ultrafast Reversal of the Ferroelectric Polarization, Phys. Rev. Lett. 118, 197601 (2017).

[13] T. Nova, A. Disa, M. Fechner, and A. Cavalleri, Metastable ferroelectricity in optically strained $\mathrm{SrTiO}_{3}$, Science 364, 1075 (2019).

[14] X. Li, T.n Qiu, J. Zhang, E. Baldini, J. Lu, A. M. Rappe, and K. A. Nelson, Terahertz field-induced ferroelectricity in quantum paraelectric $\mathrm{SrTiO}_{3}$, Science 364, 1079 (2019).
[15] D. Shin, S. A. Sato, H. Hübener, U. De Giovannini, N. Park, and A. Rubio, Dynamical amplification of electric polarization through nonlinear phononics in 2D SnTe, npj Comput. Mater. 6, 182 (2020).

[16] V. A. Abalmasov, Ultrafast reversal of the ferroelectric polarization by a midinfrared pulse, Phys. Rev. B 101, 014102 (2020).

[17] T. F. Nova, A. Cartella, A. Cantaluppi, M. Först, D. Bossini, R. V. Mikhaylovskiy, A. V. Kimel, R. Merlin, and A. Cavalleri, An effective magnetic field from optically driven phonons, Nat. Phys. 13, 132 (2017).

[18] D. M. Juraschek, M. Fechner, A. V. Balatsky, and N. A. Spaldin, Dynamical multiferroicity, Phys. Rev. Mater. 1, 014401 (2017).

[19] D. Shin, H. Hübener, U. De Giovannini, H. Jin, A. Rubio, and N. Park, Phonon-driven spin-Floquet magneto-valleytronics in $\mathrm{MoS}_{2}$, Nat. Commun. 9, 638 (2018).

[20] P. G. Radaelli, Breaking symmetry with light: Ultrafast ferroelectricity and magnetism from three-phonon coupling, Phys. Rev. B 97, 085145 (2018).

[21] S. F. Maehrlein, I. Radu, P. Maldonado, A. Paarmann, M Gensch, A. M. Kalashnikova, R. V. Pisarev, M. Wolf, P. M. Oppeneer, J. Barker, and T. Kampfrath, Dissecting spin-phonon equilibration in ferrimagnetic insulators by ultrafast lattice excitation, Sci. Adv. 4, eaar5164 (2018).

[22] M. Gu and J. M. Rondinelli, Nonlinear phononic control and emergent magnetism in Mott insulating titanates, Phys. Rev. B 98, 024102 (2018).

[23] G. Khalsa and N. A. Benedek, Ultrafast optically induced ferromagnetic/anti-ferromagnetic phase transition in $\mathrm{GdTiO}_{3}$ from first principles, npj Quantum Mater. 3, 15 (2018).

[24] M. Fechner, A. Sukhov, L. Chotorlishvili, C. Kenel, J. Berakdar, and N. A. Spaldin, Magnetophononics: ultrafast spin control through the lattice, Phys. Rev. Mater. 2, 064401 (2018).

[25] D. M. Juraschek and N. A. Spaldin, Orbital magnetic moments of phonons, Phys. Rev. Mater. 3, 064405 (2019).

[26] A. S. Disa, M. Fechner, T. F. Nova, B. Liu, M. Först, D. Prabhakaran, P. G. Radaelli, and A. Cavalleri, Polarizing an antiferromagnet by optical engineering of the crystal field, Nat. Phys. 16, 937 (2020).

[27] D. M. Juraschek and P. Narang, Shaken not strained, Nat. Phys. 16, 900 (2020).

[28] M. Rodriguez-Vega, Z.-X. Lin, A. Leonardo, A. Ernst, G. Chaudhary, M. G. Vergniory, and G. A. Fiete, Phonon-mediated dimensional crossover in bilayer $\mathrm{CrI}_{3}$, Phys. Rev. B 102, 081117(R) (2020).

[29] D. M. Juraschek, P. Narang, and N. A. Spaldin, Phono-magnetic analogs to opto-magnetic effects, Phys. Rev. Res. 2, 043035 (2020). 
[30] D. M. Juraschek, D. S. Wang, and P. Narang, Sum-frequency excitation of coherent magnons, Phys. Rev. B 103, 094407 (2021).

[31] A. Stupakiewicz, C. S. Davies, K. Szerenos, D. Afanasiev, K. S. Rabinovich, A. V. Boris, A. Caviglia, A. V. Kimel, and A. Kirilyuk, Ultrafast phononic switching of magnetization, Nat. Phys. 17, 489 (2021).

[32] F. Giorgianni, B. Wehinger, S. Allenspach, N. Colonna, C. Vicario, P. Puphal, E. Pomjakushina, B. Normand, and Ch. Rüegg, Nonlinear quantum magnetophononics in $\mathrm{SrCu}_{2}\left(\mathrm{BO}_{3}\right)_{2}$, arXiv:2101.01189.

[33] D. Afanasiev, J. R. Hortensius, B. A. Ivanov, A. Sasani, E. Bousquet, Y. M. Blanter, R. V. Mikhaylovskiy, A. V. Kimel, and A . D. Caviglia, Ultrafast control of magnetic interactions via light-driven phonons, Nat. Mater. 20, 607 (2021).

[34] D. M. Coles, P. Michetti, C. Clark, W. C. Tsoi, A. M. Adawi, J. S. Kim, and D. G. Lidzey, Vibrationally Assisted Polariton-Relaxation Processes in Strongly Coupled OrganicSemiconductor Microcavities, Adv. Funct. Mater. 21, 3691 (2011).

[35] P. Toermoe and W. L. Barnes, Strong coupling between surface plasmon polaritons and emitters: a review, Rep. Prog. Phys. 78, 013901 (2015).

[36] K. Stranius, M. Hertzog, and K. Börjesson, Selective manipulation of electronically excited states through strong light-matter interactions, Nat. Commun. 9, 2273 (2018).

[37] J. A. Hutchison, T. Schwartz, C. Genet, E. Devaux, and T. W. Ebbesen, Modifying chemical landscapes by coupling to vacuum fields, Angew. Chem. 51, 1592 (2012).

[38] J. Flick, N. Rivera, and P. Narang, Strong light-matter coupling in quantum chemistry and quantum photonics, Nanophotonics 7, 1479 (2018).

[39] R. F. Ribeiro, L. A. Martínez-Martínez, M. Du, J. CamposGonzalez-Angulo, and J. Yuen-Zhou, Polariton chemistry: controlling molecular dynamics with optical cavities, Chem. Sci. 9, 6325 (2018).

[40] J. Flick and P. Narang, Cavity-Correlated Electron-Nuclear Dynamics from First Principles, Phys. Rev. Lett. 121, 113002 (2018).

[41] A. Thomas, L. Lethuillier-Karl, K. Nagarajan, R. M. A. Vergauwe, J. George, T. Chervy, A. Shalabney, E. Devaux, C. Genet, J. Moran, and T. W. Ebbesen, Tilting a ground-state reactivity landscape by vibrational strong coupling, Science 363 , 615 (2019).

[42] F. P. Laussy, A. V. Kavokin, and I. A. Shelykh, ExcitonPolariton Mediated Superconductivity, Phys. Rev. Lett. 104, 106402 (2010).

[43] M. A. Sentef, M. Ruggenthaler, and A. Rubio, Cavity quantum-electrodynamical polaritonically enhanced electronphonon coupling and its influence on superconductivity, Sci. Adv. 4, eaau6969 (2018)

[44] F. Schlawin, A. Cavalleri, and D. Jaksch, Cavity-Mediated Electron-Photon Superconductivity, Phys. Rev. Lett. 122, 133602 (2019).

[45] J. B. Curtis, Z. M. Raines, A. A. Allocca, M. Hafezi, and V. M. Galitski, Cavity Quantum Eliashberg Enhancement of Superconductivity, Phys. Rev. Lett. 122, 167002 (2019).

[46] A. Subedi, A. Cavalleri, and A. Georges, Theory of nonlinear phononics for coherent light control of solids, Phys. Rev. B 89, 220301(R) (2014).
[47] A. von Hoegen, R. Mankowsky, M. Fechner, M. Först, and A. Cavalleri, Probing the interatomic potential of solids with strong-field nonlinear phononics, Nature (London) 555, 79 (2018).

[48] M. Fechner and N. A. Spaldin, Effects of intense optical phonon pumping on the structure and electronic properties of yttrium barium copper oxide, Phys. Rev. B 94, 134307 (2016).

[49] R. Mankowsky, M. Först, T. Loew, J. Porras, B. Keimer, and A. Cavalleri, Coherent modulation of the $\mathrm{YBa}_{2} \mathrm{Cu}_{3} \mathrm{O}_{6+x}$ atomic structure by displacive stimulated ionic Raman scattering, Phys. Rev. B 91, 094308 (2015).

[50] R. Mankowsky, M. Fechner, M. Först, A. von Hoegen, J. Porras, T. Loew, G. L. Dakovski, M. Seaberg, S. Möller, G. Coslovich, B. Keimer, S. S. Dhesi, and A. Cavalleri, Opticallyinduced lattice deformations, electronic structure changes, and enhanced superconductivity in $\mathrm{YBa}_{2} \mathrm{Cu}_{3} \mathrm{O}_{6.48}$, Struc. Dyn. 4, 044007 (2017).

[51] D. M. Juraschek and S. F. Maehrlein, Sum-frequency ionic Raman scattering, Phys. Rev. B 97, 174302 (2018).

[52] A. A. Melnikov, K. N. Boldyrev, Yu. G. Selivanov, V. P. Martovitskii, S. V. Chekalin, and E. A. Ryabov, Coherent phonons in a $\mathrm{Bi}_{2} \mathrm{Se}_{3}$ film generated by an intense single-cycle THz pulse, Phys. Rev. B 97, 214304 (2018).

[53] B. E. Knighton, R. T. Hardy, C. L. Johnson, L. M. Rawlings, J. T. Woolley, C. Calderon, A. Urrea, and J. A. Johnson, Terahertz waveform considerations for nonlinearly driving lattice vibrations, J. Appl. Phys. 125, 144101 (2019).

[54] C. L. Johnson, B. E. Knighton, and J. A. Johnson, Distinguishing Nonlinear Terahertz Excitation Pathways with Two-Dimensional Spectroscopy, Phys. Rev. Lett. 122, 073901 (2019).

[55] A. A. Melnikov, Yu. G. Selivanov, and S. V. Chekalin, Phonondriven ultrafast symmetry lowering in $\mathrm{Bi}_{2} \mathrm{Se}_{3}$ crystal, Phys. Rev. B 102, 224301 (2020).

[56] M. A. Sentef, Light-enhanced electron-phonon coupling from nonlinear electron-phonon coupling, Phys. Rev. B 95, 205111 (2017).

[57] S. Fahy, É. D. Murray, and D. A. Reis, Resonant squeezing and the anharmonic decay of coherent phonons, Phys. Rev. B 93, 134308 (2016).

[58] S. W. Teitelbaum, T. Henighan, Y. Huang, H. Liu, M. P. Jiang, D. Zhu, M. Chollet, T. Sato, É. D. Murray, S. Fahy, S. O'Mahony, T. P. Bailey, C. Uher, M. Trigo, and D. A. Reis, Direct Measurement of Anharmonic Decay Channels of a Coherent Phonon, Phys. Rev. Lett. 121, 125901 (2018).

[59] D. M. Juraschek, Q. N. Meier, and P. Narang, Parametric Excitation of an Optically Silent Goldstone-Like Phonon Mode, Phys. Rev. Lett. 124, 117401 (2020).

[60] R. Merlin, Generating coherent THz phonons with light pulses, Solid State Commun. 102, 207 (1997).

[61] T. Dekorsy, G. C. Cho, and H. Kurz, Coherent phonons in condensed media, in Topics in Applied Physics, Vol. 76, edited by M. Cardona and G. Güntherodt (Springer-Verlag, Berlin, Heidelberg, 2000), pp. 169.

[62] A. Subedi, Midinfrared-light-induced ferroelectricity in oxide paraelectrics via nonlinear phononics, Phys. Rev. B 95, 134113 (2017).

[63] D. M. Juraschek, M. Fechner, and N. A. Spaldin, Ultrafast Structure Switching through Nonlinear Phononics, Phys. Rev. Lett. 118, 054101 (2017). 
[64] A. Cartella, T. F. Nova, M. Fechner, R. Merlin, and A. Cavalleri, Parametric amplification of optical phonons, Proc. Nat. Acad. Sci. USA 115, 12148 (2018)

[65] See Supplemental Material at http://link.aps.org/supplemental/ 10.1103/PhysRevResearch.3.L032046 for a derivation of the coupled equations of motion for the phonon and cavity modes.

[66] Note that for very strong driving, the mode effective charge can be dependent on the phonon amplitude [64].

[67] D. Werdehausen, T. Takayama, M. Höppner, G. Albrecht, A. W. Rost, Y. Lu, D. Manske, H. Takagi, and S. Kaiser, Coherent order parameter oscillations in the ground state of the excitonic insulator $\mathrm{Ta}_{2} \mathrm{NiSe}_{5}$, Sci. Adv. 4, eaap8652 (2018).

[68] A. Zong, X. Shen, A. Kogar, L. Ye, C. Marks, D. Chowdhury, T. Rohwer, B. Freelon, S. Weathersby, R. Li, J. Yang, J. Checkelsky, X. Wang, and N. Gedik, Ultrafast manipulation of mirror domain walls in a charge density wave, Sci. Adv. 4, eaau5501 (2018).

[69] M. Rodriguez-Vega, M. Vogl, and G. A. Fiete, Direct driving of electronic and phononic degrees of freedom in bilayer graphene with infrared light, arXiv:2011.05089.
[70] A. von Hoegen, M. Fechner, M. Först, J. Porras, B. Keimer, M. Michael, E. Demler, and A. Cavalleri, Probing coherent charge fluctuations in $\mathrm{YBa}_{2} \mathrm{Cu}_{3} \mathrm{O}_{6+x}$ at wavevectors outside the light cone, arXiv: 1911.08284

[71] P. Sivarajah, A. Steinbacher, B. Dastrup, J. Lu, M. Xiang, W. Ren, S. Kamba, S. Cao, and K. A. Nelson, THz-frequency magnon-phonon-polaritons in the collective strong-coupling regime, J. Appl. Phys. 125, 213103 (2019).

[72] Y. Ashida, A. Imamoglu, J. Faist, D. Jaksch, A. Cavalleri, and E. Demler, Quantum Electrodynamic Control of Matter: Cavity-Enhanced Ferroelectric Phase Transition, Phys. Rev. X 10, 041027 (2020).

[73] S. Latini, D. Shin, S. A. Sato, C. Schäfer, U. De Giovannini, H. Hübener, and A. Rubio, The ferroelectric photo-groundstate of $\mathrm{SrTiO}_{3}$ : Cavity materials Engineering, PNAS 118, e2105618118 (2021).

[74] A. Völkel and G. Herink, Terahertz phonons coherently couple ultrashort solitons inside a cavity, arXiv:2104.01691. 